\title{
Efficient Resource Discovery in Mobile Ad Hoc Networks
}

\author{
Ravi Thanawala and Jie Wu \\ Department of Computer Science and Engineering \\ Florida Atlantic University \\ Boca Raton, FL 33431 \\ Email: \{rthanawa@,jie@cse.\}fau.edu
}

\author{
Avinash Srinivasan \\ Mathematics, Computer Science, and Statistics \\ Bloomsburg University of Pennsylvania \\ Bloomsburg, PA 17815 \\ Email: avinash@bloomu.edu
}

\begin{abstract}
The highly dynamic nature of infrastructureless adhoc networks poses new challenges during resource discovery. In this paper, we propose a novel algorithm for resource discovery in mobile ad hoc networks called Efficient Resource-Discovery (ERD). When proposing this novel algorithm, our primary goal is to spread the most relevant resources and queries to the nodes in the network. The proposed algorithm ERD is very efficient in dynamically ranking resources and queries based on their priority, selecting the transmission time, and determining how many resources and queries are to be transmitted. ERD utilizes the network bandwidth in an optimal manner avoiding the spread of redundant data in the network, which otherwise can significantly overload the network with duplicate copies. We compare ERD with periodic flooding and rank based broadcast (RBB) algorithms for mobile ad hoc networks. Results show that ERD outperforms both these algorithms significantly.
\end{abstract}

Index Terms - Resource discovery, mobile ad hoc networks (MANET), mobility, simulation

\section{INTRODUCTION}

A mobile ad hoc network (MANET) is a network in which nodes exchange messages with each other through unregulated short-range wireless technologies such as IEEE 802.11 and Bluetooth. Resource discovery, particularly, is critical in the design of a MANET where nodes do not have any prior knowledge of the available resources in the network.

Searching for information is a very challenging task in MANET because of the unpredictable mobility of nodes. As nodes travel around the network, they continually establish several ephemeral connections with other peers along the way and exchange necessary messages with their neighbors. For instance, a driver who is looking for a parking space spreads a request throughout the network until he gets information about an available space. To tackle such a situation in an optimized way, we introduce the Efficient Resource-Discovery (ERD) algorithm for searching resources in the network. We refer to the data requested by a node in the network as Resources. In $\mathrm{ERD}$, a moving node disseminates the request for a resource (queries) and the resource availability information in the network. The algorithm efficiently spreads the information request in the network without overloading it and searches for a node that has the requested resource.
In resource discovery, there are two approaches for searching the resource: the push approach and the pull approach [1][5][7]. In the push approach, resources are pushed through the network so that they reach nodes that have requested the resources. In the pull approach, a node floods the network with a resource request. Upon finding the node which has the requested resource, a routing path is created to connect the resource to the request originator. Our algorithm is a hybrid between the push and pull approaches. In ERD, when a moving node $A$ comes in contact with another node $B$, they exchange a list of queries and resources that have high priority in the network. Node $B$ updates its database as soon as it receives the list from node $A$. Later, when node $B$ comes in contact with other nodes, it repeats the process of spreading high-priority data. Consequently, important queries and resources are spread among nodes across the network.

Resource discovery in MANETs is a challenging problem for three primary reasons. First - due to the dynamic nature of the network, there is no proper knowledge about the availability of resources and the locations of nodes. The nodes have to search for this information, which is difficult with nodes moving at different speeds in different directions. Second message duplication is another major concern while disseminating messages. A node can discard a duplicate message, but this leads to problems concerning bandwidth usage. The spread of redundant data reduces the efficiency of spreading messages in the network and, as a result, the performance suffers. In ERD, the spread of duplicate messages is avoided to ensure efficient utilization of network bandwidth. Third memory of nodes is limited regarding where messages are stored. As a node moves through the network, it continues to receive new messages from other nodes and deletes older messages from its memory. There is a high probability that some high-priority messages will be lost in this process. The strategy of ERD is to disseminate a list of important queries and resources in the network that keeps nodes updated on the latest requirements of the network at any given point in time.

The remainder of the paper is organized as follows: Section II lists related. Section III explains our algorithm. Section IV presents simulation results. Section V concludes the paper with directions for future research. 


\section{RELATED WORK}

Flooding [8] is a common technique that is used to search data in the network. In [3], Yuan and $\mathrm{Wu}$ discuss a publish/subscribe protocol that delivers events to interested nodes in the network. The Rank-Based Broadcast (RBB) technique [2] is another resource discovery technique, which ranks resources in its database and broadcasts the top-ranked resources to its neighbors. Zhao, Ammar and Zegura [4] introduce a technique using a message ferrying approach.

TACO-DTN [11], a content dissemination system, which by virtue is time-aware in terms of subscriptions and events, is appropriate for delay tolerant networks. In this system, there are nodes which act as infostations [10] that are located at specific positions where there are high chances of interested subscribers moving. The self-limiting epidemic forwarding protocol [9] is another dissemination technique which is defined around the local scope of the node. The authors present a scheme where the forwarding of messages is controlled by manipulating the time to live (TTL). This protocol is broadcast in nature, but there is some control over the spread of messages in the network.

\section{EFFICIENT RESOURCE-DISCOVERY (ERD) ALGORITHM}

Our model consists of a fixed number of moving nodes which move in a finite geographic space. These nodes have a specific transmission range and fixed memory size. The nodes move according to the random way-point mobility model.

Queries, Resources, and Acknowledgments: Every node periodically exchanges queries, resources, and acknowledgments with its neighbors. A query $q_{i}$ consists of (1) a unique identifier $i$, (2) the name of the source that initiated the query, (3) the name of the requested resource, (4) the number of hops that it has traveled, (5) the time it was issued $t_{i}$ at, and (6) a signature. The resource name can be of any type or specification, but for simplicity we assume it to be a particular name like a file name or specific data. Resource $r_{j}$ is the data which a node is looking for in the network. Resources disseminated by a node are based on the queries it has.

Hello messages are another prime component of our algorithm. They consist of a node identifier ( id ) and an acknowledgment list. The acknowledgment list consists of (1) (nid) of the node that has requested the query $q_{i}$, (2) name of the query $q_{i}$ and (3) timer $T_{i}$ for the acknowledgment. An acknowledgement list is represented as follows: $\left(\left[n_{1}, q_{1}, T_{1}\right],\left[n_{2}, q_{2}, T_{2}\right],\left[n_{3}, q_{3}, T_{3}\right], \ldots\right)$, etc. The acknowledgments in this list are discarded once their life time expires. Our algorithm executes in four distinct phases: a) Neighbor detection, b) Dynamic ranking of the queries, c) Dynamic ranking of the resources, and d) Dissemination.

\section{A. Neighbor detection}

A node periodically exchanges a Hello message with its neighbors. The Hello message contains the node identifier (nid) and the acknowledgment list. At the end of this phase,

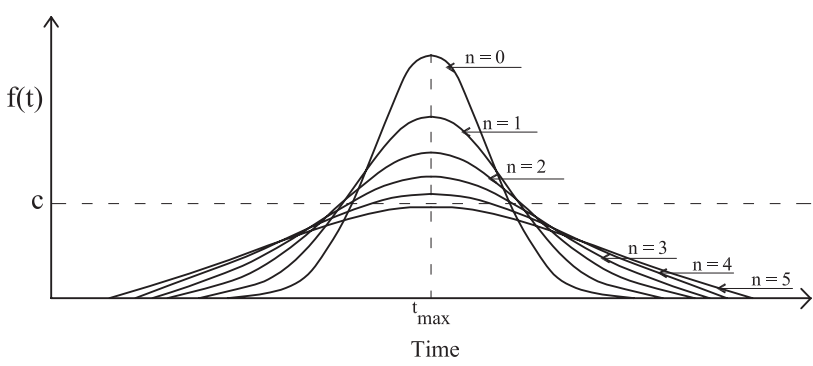

Fig. 1. Ranking function graph using the Gaussian function.

the node knows its neighbors and has updated information about the requirements of the neighboring nodes.

\section{B. Dynamic ranking of the queries}

At this stage, the node has queries which are not yet serviced and they need to be spread in the network. The algorithm chooses a small amount of queries which are important at that point in time to avoid spreading duplicate queries in the network and to stay within the limits of the bandwidth constraint. Queries are dynamically ranked and the top-ranked queries are considered for dissemination. The ranking is done such that duplicate data is not spread in the network and a significant number of nodes do not have that query. Time $t_{i}$ is the time at which the query was initiated. This variable is used to determine the rank of the query $q_{i} . t_{i}$ also indicates how long the query has been traveling in the network. The greater the value of $t_{i}$, the less important the query is because it indicates that the query is relatively old and could have been sent to many nodes. A lower value of $t_{i}$ indicates that the query is relatively new and should be spread quickly. Dynamic ranking of the queries is done using the following function:

$$
f(t)=\frac{1}{\delta \sqrt{2 \pi}} e^{-\frac{(t-\mu)^{2}}{2 \delta^{2}}}
$$

where, $f(t)$ is the ranking function for queries, and $t$ is the time when the query was initiated. $\delta$ and $\mu$ are constants used to determine the maximum time and threshold time respectively. The Gaussian function is used to find the ranking function of the queries. The nature of the Gaussian equation gives the queries the pattern in which its importance can be determined while ranking. This ranking function is shown in Figure 1 and it displays the graph for different values of $\delta$.

In the graph, we vary values of $\delta$ to represent different values $n$, the number of hops. In the figure, the Y-axis has the ranking function and the $\mathrm{X}$-axis represents the lifetime of the query. There is a cut-off limit $c$ for the ranking function for every value of $n$. The cut-off value $c$ is used when disseminating queries, which we discuss later in the paper. Every graph has a $t_{\max }$, where the ranking function $f(t)$ is the maximum for that particular value of $n$. Ranking of queries is done with respect to time, in a special manner. According to the above function, when a query is initiated, it has a low level of importance; gradually over time, its importance increases. Consider that query $q_{1}$ has $n=0$ when initiated. The rank of 
this query is lower as the value of the ranking function $f(t)$ is less when the query is initiated. Queries that are generated before $q_{1}$ are of more importance because they need to be disseminated in the network first. Gradually, the importance of $q_{1}$ increases with respect to time and its rank rises. Thus, the chances of $q_{1}$ being transmitted are higher when its lifetime is around $t_{\max }$.

When a query reaches $t_{\max }$, its importance starts decreasing because it is assumed that there will be enough copies of that query in the network by this time. Hence, the probability of $q_{1}$ being spread starts decreasing as its $f(t)$ starts decreasing. After a threshold lifetime of the query, it is discarded from the nodes' local database. In Figure 1, there is a different graph for different values of $n$. A query which has traversed through fewer nodes is considered to have higher priority and consequently a higher value of $f(t)$ when compared to the query which has traversed through more nodes. For instance, assume there are two queries with a node- $q_{1}$ that has $n=0$ and $q_{2}$ that has $n=3$. This indicates that $q_{2}$ has traveled in the network more than $q_{1}$ and there is a higher probability that $q_{2}$ has been spread to more nodes. So, while ranking queries, $q_{1}$ should be given a higher priority over $q_{2}$. Because of this, we have higher $f\left(t_{\max }\right)$ for a query which has traveled to fewer nodes. After ranking the queries using the ranking function, the queries are once again scanned using the queries' signatures to re-rank the queries. Every query has a signature containing the list of nodes containing it.

A query $q_{1}[\mathrm{~A}, \mathrm{~B}, \mathrm{C}]$ shows that nodes $A, B$ and $C$ have query $q_{1}$ in their local database. Every node has the node ID of its neighbors that are received along with the Hello message. Queries which are already with the neighbors are not transmitted to preserve precious bandwidth and avoid transmission of duplicate copies. This procedure is explained in Figure 2. There are two nodes, $A$ and $B$, in each other's transmission range, with their own query list in their local database. Suppose node $A$ has to transmit its top 2 queries from $q_{1}, q_{2}$ and $q_{3}$, which are shown with their respective signatures in Figure 2. The signature of $q_{1}$ shows that nodes $A$ and $B$ have it. If $q_{1}$ is transmitted to $B$, then it will receive another copy of the same query and will discard it. This can be avoided by not sending $q_{1}$ to $B . q_{1}$ is then pushed to a lower rank in the list instead, and $q_{2}$ and $q_{3}$ are considered to be transmitted to $B$. At the end of this phase, queries are ranked using the ranking function and the queries' signatures, as discussed previously.

\section{Dynamic ranking of the resources}

Resources are ranked using queries that were ranked in the previous phase; the ranking itself is done as follows:

$$
f\left(d_{i}\right)=\frac{\# n\left(d_{i}\right)}{\#\left(d_{i}\right)}
$$

where, $d_{i}$ is the resource or data (resource information), $\# n\left(d_{i}\right)$ is the number of nodes requesting $d_{i}$, which can be determined from the queries that are ranked in the previous
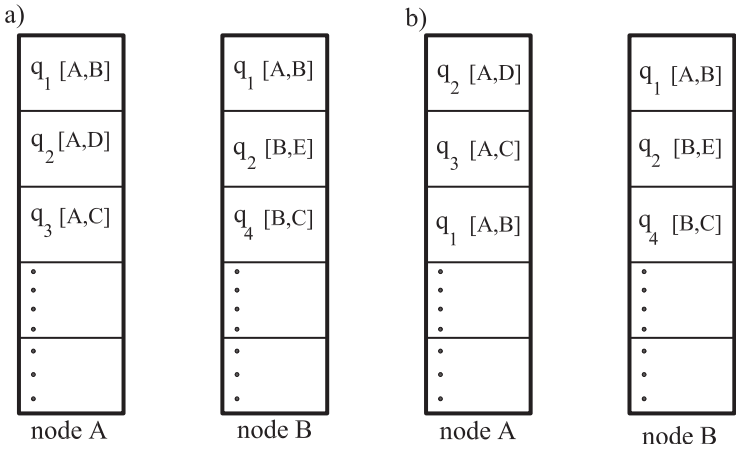

Fig. 2. State of the queries with their signatures (a) before the exchange of queries (b) after the exchange of queries.

step, and $\#\left(d_{i}\right)$ is the number of nodes having the resource $d_{i}$, which can be determined from the signature of the resource. Every resource has a signature attached to it which indicates how many nodes have that particular resource. For instance, $r_{1}[\mathrm{~A}, \mathrm{D}, \mathrm{E}]$ is a resource which is held by nodes $A, D$ and $E$. A higher value of $\# n\left(d_{i}\right)$ indicates that too many nodes have requested that resource and very few nodes have it.

\section{Dissemination}

The objective of our algorithm is to spread resources based on queries that have high priority in the network at that point in time. In previous phases, we determined high priority queries and resources, which are dynamically ranked by nodes. The top-ranked queries and resources need to be transmitted to their direct neighbors. A major concern while disseminating queries and resources is the bandwidth. As bandwidth is limited, it needs to be distributed between resources and queries efficiently. ERD makes the decision of allocating bandwidth for the transmission of queries and resources dynamically. The value of $f(t)$ [Eq. 1] and $f\left(d_{i}\right)$ [Eq. 2] of the query and resource respectively, are used in allocating the bandwidth. The list of queries whose $f(t)$ is greater than the cut-off limit $c$, as in Figure 1, are considered for dissemination. In the remainder of this paper, we will refer to this cut-off $c$ as bandwidth $h_{\text {query. }}$. Queries are then sorted based on $f(t)$. Similarly, for resources, bandwidth $h_{\text {resource }}$ is the threshold value for the resources. Resources with $f\left(d_{i}\right)$ greater than bandwidth $h_{\text {resource }}$ are considered ready for transmission. We assume that queries and resources above these cut-off values are new in the network and need to be transmitted sooner. Although the query or resource is new, it's transmission is decided based on the bandwidth available. It might be transmitted in future transmissions.

However, before transmission, the allocated bandwidth is considered to decide how many queries and resources can be transmitted. Suppose the allocated bandwidth allows the transmission of 6 queries and 9 resources which sums up to 15 data packets that need to be transmitted. Consider a scenario where there are 8 queries with $f_{t}$ above bandwidth $h_{\text {query }}$ and 12 resources whose $f\left(d_{i}\right)$ is above bandwidth $h_{\text {resource }}$. Then, only the top 6 queries and the top 9 resources are transmitted 
a)

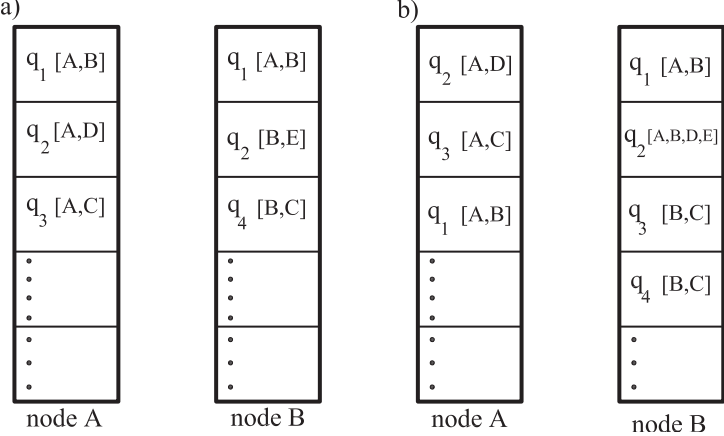

Fig. 3. State of the queries with their signatures (a) before exchange of the queries (b) merging of queries after exchange.

based on the available bandwidth. Consider another special case, where there are only 4 queries whose $f(t)$ values are above the bandwidth $h_{\text {query }}$ and there are 13 resources with

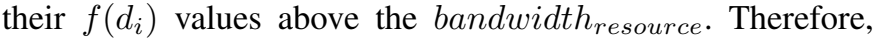
from the sorted list of queries, we can see that there are only 4 queries which are new. Out of the 13 resources which qualify for transmission, only the top 11 resources are selected. Finally, the node transmits 4 queries and 11 resources, which sums up to 15 data packets. This way the bandwidth will be distributed dynamically between queries and resources, based on requirement. The bandwidth is shared in an optimized way and important data is spread in the network.

Once queries are transmitted to neighbors, merging queries' signatures is another important feature of our ERD algorithm. Nodes are not aware of which queries are stored in other nodes. For instance, in Figure 3, the signature of $q_{2}$ in node $A$ shows that $q_{2}$ is in nodes $A$ and $D$, whereas node $B$ shows that $q_{2}$ is in nodes $B$ and $E$. From this, it can be concluded that $q_{2}$ is in nodes $A, B, D$ and $E$. However, $A$ and $B$ are not aware of this complete information. To solve this problem, when $q_{2}$ is received by node $B$, the queries' signatures are merged at $B$. In Figure 3, the signature merge of $q_{2}$ from nodes $A$ and $B$ is $[A, B, D, E]$. During the future spread of $q_{2}$, the merged signature will be considered so that will reduce the spread of redundant data in the network will be reduced.

In our proposed algorithm ERD, we assume that nodes have a limited memory and that nodes cannot afford to lose important data that is important in the network at that point in time. Therefore, when the memory limit is reached, queries and resources of lower ranks from the ranked list are discarded to make space for new data. In this way, ERD ensures that important data is not deleted from nodes' local database.

\section{Simulation \& Results}

\section{A. Simulation Environment}

In our simulation, mobile nodes move in the network and spread resources and queries. We adopt the random waypoint mobility model in our simulation study. Each node moves in a $0.5 \times 0.5$ mile square area with a random speed and in a random direction. Each node has a fixed lifetime after which it perishes from the network. The lifetime of each node

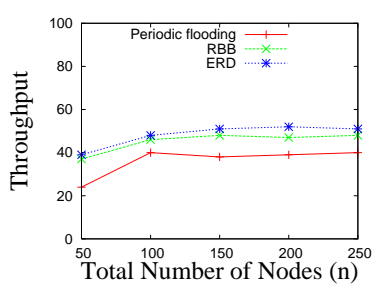

(a) Range $=50 \mathrm{mts}$

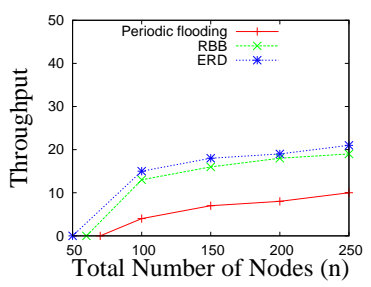

(b) Range $=10 \mathrm{mts}$

Fig. 4. Throughput vs. Density of the network.

is randomly chosen from [100 - 600] time units, before the start of the algorithm. The whole simulation runs for 1,000 time units. Each node can hold up to 45 resources and these resources are essentially numbers from $[0,45]$. Similarly, each node can hold up to 40 queries selected from [0, 40]. For our simulation, $t_{\max }=60$ seconds, where $f(t)$ is the maximum i.e. 0.1 . We consider the cut-off value $C$ to be equal to 0.06 .

The performance measure function we consider in our simulation is the throughput, which is the average number of matching resource messages received by a moving object.

\section{B. Simulation Results}

In periodic flooding, a node does not dynamically select the flooding period based on the network condition. Therefore, the flooding period can either be too long, which can leave the network in an idle state, or too short in which case the traffic in the network will be very high. In the RBB technique, resources are ranked and the top-ranked resources are broadcast. During every broadcast, only the native query of the node is transmitted and other queries in the local database of the node are not spread. In RBB, native queries are transferred only when nodes come in direct contact with each other. This reduces the spread of queries. In our model, both queries and resources are ranked prior to transmission. The transmission period is decided on the basis of new neighbors. The dynamic ranking of queries and resources on the basis of the current demand in the network spreads the most promising data in the network.

The results are shown in Figure 4(a), where the number of nodes is varied and the throughput is noted for each of the three algorithms- periodic flooding, ERD, RBB. It can be observed that as the number of nodes increases, the throughput also increases. In the graph, ERD performs better than both periodic flooding and RBB. The performance of periodic flooding is the worst because redundant resources are broadcasted in the network. Hence, the throughput is affected since nodes do not receive their requested resource. The performance of RBB is better than periodic flooding, because only the native query is being transmitted along with the topranked resources. In Figure 4(b), we compare the performance by reducing the transmission range of nodes to 10 meters. With a smaller transmission range, the node comes in contact with fewer neighboring nodes thereby resulting in less resources and queries being spread. Consequently, there is a decline in the performance when the transmission range is reduced. 


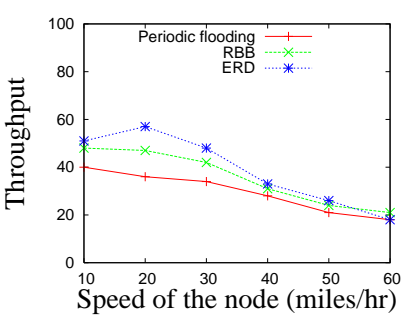

(a) Range $=50 \mathrm{mts}$

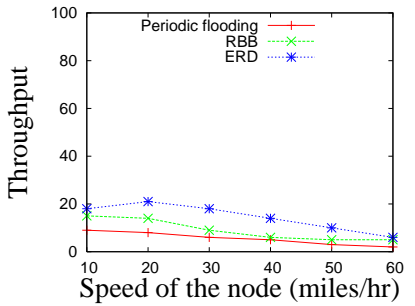

(b) Range $=10 \mathrm{mts}$
Fig. 5. Throughput vs. Speed of the nodes.

Figures 5(a) and 5(b) show the performance of each algorithm as the node speed is varied. The transmission range of the node is fixed at 50 meters in Figure 5(a). According to the results, as the speed increases, the performance declines as the time spent by a node in the transmission range of another node is reduced. As the speed decreases, nodes remain in each others transmission range for a longer time period and hence, can exchange messages. Therefore, the performance is better initially, but when the speed is increased, the time spent by a node in the transmission range of another node is less. In Figure 5(b), we reduce the transmission range to 10 meters and we can see that ERD gives better results. However, the overall performance declines because the node comes across relatively fewer neighbors during movement in the network when the transmission range is shorter.

In Figure 6(a), we vary the bandwidth allocated to nodes and check the performance of the algorithms. The greater the number of messages transmitted, the higher the chances that the node gets its required resources. In RBB, as bandwidth increases, so does the broadcast of the number of resources in the network. ERD outperforms RBB because it also spreads queries along with its native query. Figure 6(b) shows that ERD drastically decreases the number of query messages transferred during a search. The ERD manages to keep message transfers almost at a fixed level as the decision is taken dynamically before every transmission. Thus, by using the network bandwidth efficiently, ERD is able to scale well as network size increases.

\section{Simulation summary}

From the above simulation results, it can be observed that the performance of our ERD algorithm is better than the flooding algorithm and the RBB technique. The dynamic ranking of queries and resources by ERD gives better throughput. The spread of top queries helps to inform the requirements of other nodes in the network. ERD utilizes the bandwidth in an optimized manner, which results in better performance. Nodes will dynamically decide how many queries and resources need to be transmitted based on their importance. This results in spreading only the important data in the network. The performance of ERD is better than both periodic flooding and the RBB algorithm as it transmits more important data based on the overall requirement of the network. Hence, ERD gives

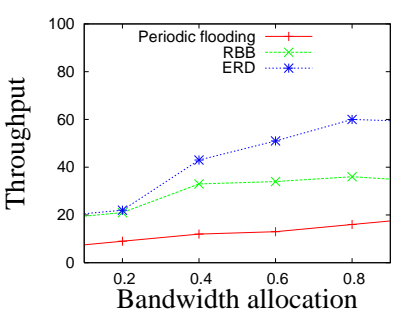

(a) Bandwidth

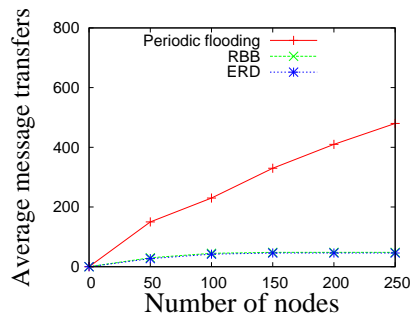

(b) Message transfers
Fig. 6. Studying the optimal behavior of ERD.

optimal results in dense networks and utilizes the bandwidth effectively and efficiently.

\section{CONCLUSIONS}

In this paper, we proposed ERD, an algorithm which optimally disseminates queries and resources in the network without spreading redundant data. Nodes in ERD dynamically select queries and resources, which are transmitted to their neighbors. This decision is made based on novel techniques for prioritizing and disseminating mobile information, as well as novel techniques for effective bandwidth utilization in mobile data dissemination. Important queries and resources are given more preference discarding less important ones from a node's memory. We compared the ERD algorithm to periodic flooding and RBB algorithms; results show that ERD significantly outperforms the other two algorithms. These results show lot of promise for the efficient resource discovery algorithm. We plan to further evaluate this algorithm under various scenarios of mobility patterns and resource distribution.

\section{REFERENCES}

[1] Y. Huang and H. Garcia-Molina. Publish/subscribe in a mobile environment. Proc. of ACM MoBiDE, 2001.

[2] O. Wolfson, B. Xu, H. Yin and H. Cao. Search-and-discover in mobile P2P network databases, Proc. of IEEE ICDCS, 2006.

[3] Q. Yuan and J. Wu. DRIP: A dynamic voronoi regions-based publish/subscribe protocol in mobile networks. Proc. of IEEE INFOCOM, 2008

[4] W. Zhao, M. Ammar and E. Zegura. A message ferrying approach for data delivery in sparse mobile ad hoc networks. Proc. of ACM MOBIHOC, 2004.

[5] Y. Huang and H. Garcia-Molina. Publish/subscribe tree construction in wireless ad-hoc networks. Proc. of MDM, 2003.

[6] A. Vahdat and D. Becker. Epidemic routing for partially connected ad hoc networks. Technical Report CS-2000-06, Department of Computer Science, Duke University, 2000.

[7] R. Zhang and Y. Hu. HYPER: a hybrid approach to efficient content-based publish/subscribe. Proc. of IEEE ICDCS, 2005.

[8] R. Oliveira, L. Bernardo, and P. Pinto. Flooding techniques for resource discovery on high mobility MANETs. Proc. of IWWAN, 2005.

[9] A. E. Fawal, J. V. Le Boudec and K. Salamatian. Self-limiting epidemic forwarding. Technical report Ref. LCA-REPORT-2006-126, 2006.

[10] R. H. Frenkiel, B. R. Badrinath, J. Borres and R. D. Yates. The infostations challenge: balancing cost and ubiquity in delivering wireless data. IEEE Wireless Communications, 7(2):6671, 2000.

[11] G. Sollazzo, M. Musolesi and C. Mascolo. TACO-DTN: a time-aware content-based dissemination system for delay tolerant networks. Proc. of ACM MobiOpp, 2007.

[12] H. Takagi and L. Kleinrock. Optimal transmission ranges for randomly distributed packet radio terminals. Trans. IEEE on Commun, 1984. 